\title{
Treatment of the hyperuricemia of gout in the United States
}

Recent developments underlying re-emergence of chronic gout management as a topic of interest include: increased incidence/prevalence of gout, especially among persons with renal, $\mathrm{CV}$, and metabolic comorbidities; suboptimal gout patient outcomes due to failure to maintain subsaturating serum urate levels (sUA); and increasing mechanistic understanding of hyperuricemia and urate crystal-induced inflammation. Novel therapeutic initiatives for controlling chronic progressive and destructive features of gout have accompanied renewed interest. Urate-lowering therapy (ULT) remains the backbone of chronic gout treatment, and substantial evidence supports dose-titrating ULT to goal sUA $<6.0$ $\mathrm{mg} / \mathrm{dL}$. (Currently, treatment of asymptomatic hyperuricemia is not recommended in the US.) The xanthine oxidase (XO) inhibitor, allopurinol, remains the most commonly prescribed ULT but achieves goal sUA in $<50 \%$ of patients when prescribed at $\leq 300 \mathrm{mg} /$ day $\quad$ (the case in $\sim 95 \%$ of patients) . Febuxostat, a structurally distinct ULT, selectively inhibits XO by a mechanism differ-
Michael A. Becker

ing from that of allopurinol and was introduced in 2009. UL efficacy is comparable with febuxostat $40 \mathrm{mg}$ /day and allopurinol $300 \mathrm{mg} /$ day, but with febuxostat 80 $\mathrm{mg}$ /day UL goal achievement is superior, with no difference in safety profile. In contrast to allopurinol, febuxostat dose reduction in mild or moderate CKD is unnecessary. Probenecid, the only potent uricosuric agent available in the US is rarely $(\sim 3 \%)$ used due to poor UL efficacy in CKD, multiple daily dosing, and drug interactions. The potent biological agent pegloticase, a PEGylated mammalian recombinant uricase that converts urate to allantoin, is approved for treatment of advanced gout refractory to conventional oral agents. Newer uricosuric agents (RDEA 594, trinilast, MBX102) and the purine nucleoside phosphorylase inhibitor BCX4208 are in clinical development either as UL monotherapy or combined with an XO inhibitor. Availability of mechanistically distinct ULTs should enhance control of hyperuricemia and consequently of clinical signs and symptoms across the spectrum of chronic gout patients.

The University of Chicago 